\title{
Improved Chamfer Matching Using Interpolated Chamfer Distance and Subpixel Search
}

\author{
Tai-Hoon Cho \\ School of Information Technology, Korea University of Technology and Education, \\ 307 Gajun-ri, Byungchun-myun, Chonan, Choongnam, Korea \\ thcho@kut.ac.kr
}

\begin{abstract}
Chamfer matching is an edge based matching technique that has been used in many applications. The matching process is to minimize the distance between transformed model edges and image edges. This distance is usually computed at the pixel resolution using a distance transform, thus reducing accuracy of the matching. In this paper, an improved approach for accurate chamfer matching is presented that uses interpolation in the distance calculation for subpixel distance evaluation. Also, instead of estimating the optimal position in subpixel using a neighborhood of the pixel position with the minimum distance, for more accurate matching, we use the Powell's optimization to find the distance minimum through actual distance evaluations in subpixel. Experimental results are presented to show the validity of our approach.
\end{abstract}

\section{Introduction}

Object matching, finding objects belonging to one image in another image, is a very important problem in computer vision and image analysis. It is particularly useful for industrial applications, where a model of an object must be aligned with an image of the object. The transformation or pose obtained by this object matching process can be used for various tasks, e.g., automatic pose adjustment in pick and place operations. In most cases, the model of the object is generated from an image of the object.

There are roughly two approaches in object matching: image-based matching and feature-based matching. Feature-based matching uses features, e.g., edges or corners, extracted from the image and the model, instead of using gray values directly. Perhaps edges are the most important low-level features. The edge based matching is more robust than the image based matching under non-uniform illumination conditions that can occur in typical industrial applications. Also, since edges in an image are a more compact representation than the image itself, the edge-based matching is computationally more efficient than the image-based matching in general.

A notable approach among edge-based matching approaches is chamfer matching, which was first proposed by Barrow et al. [1], and further improved in [2]. Since then it has been extensively used in many applications, e.g., [3][4][5]. The matching process is minimization of the distance between transformed model edges and image edges. One critical problem of this approach is that calculation of the distance between the transformed model edges and the image edges is usually done at the pixel 
resolution using a distance transform, thus reducing accuracy of the matching. With pixel-level distance computation, even use of a subpixel algorithm for the matching will not improve much the accuracy due to insensitivity to subpixel variation.

In this work, an improved approach for accurate chamfer matching is presented that uses interpolation in the distance calculation for subpixel distance evaluation. For more accurate matching, instead of estimating the optimal position in subpixel using the neighborhood of the pixel position with minimum distance, we use an optimization method like Powell's [6] that can find the minimum of a function through actual function evaluations in subpixel. Experimental results are presented to show the validity of our approach.

\section{Chamfer Matching}

\subsection{Overview}

Chamfer matching [2] is a technique that can recognize an object via 2-dimensional edge contour matching. The edge contour image of a known object is called "prepolygon image", and the edge contour image of the image, within which the object is searched, is called "predistance image". The edge contour image is a binary image consisting of edges extracted by an edge detection operator.

In the predistance image, each non-edge pixel is given a distance value from nearest edge pixel. Computation of the true Euclidean distance requires excessive time and memory resources, therefore an approximation is desirable. The operation converting a binary image to an approximate distance image is called a distance transformation (DT). The DT used in the matching algorithm should be a reasonably good approximation of the Euclidean distance; otherwise the discriminating power of the matching measure, computed from the distance values, becomes poor. It is also desirable that global distances in the image are approximated by propagating local distances (distances between neighboring pixels) over the image.

The 3-4 DT, a common choice for the DT, uses 3 for the distance between horizontal/vertical neighbors and 4 for the distance between diagonal neighbors in the $3 \times 3$ neighborhood. The 3-4 DT is known to have maximum difference of $8 \%$ compared with the Euclidean distance, thus being a good approximation of the Euclidean distance [2]. Since edge points are influenced by noise, computing the exact Euclidean distance is usually not necessary. For a more accurate DT than the 3-4 DT, the 5-7-11 DT [11] based on the $5 \times 5$ neighborhood could be used, although its computation should be more complex.

The distance image based on the DT can be obtained very efficiently by a sequential DT algorithm [2]. In the binary edge image, each edge pixel is first set to zero, and each non-edge pixel is set to infinity. Then, two passes are made over the image, first "forward" in the raster scan order (from left to right and from top to bottom), and then "backward" in the reverse raster scan.

In the prepolygon image edge pixels are extracted and converted to a list of $(\mathrm{x}, \mathrm{y})$ coordinate pairs, where $\mathrm{x}$ and $\mathrm{y}$ represents column and row number, respectively. 
From this list the edge points that are actually used for matching are chosen. The list of selected points is called the "polygon".

When the polygon is superimposed on the distance image, an average of the pixel values of the distance image at the polygon coordinates is the measure of correspondence between the edges, called the "edge distance". A perfect fit between the two edges will yield edge distance zero since each polygon point corresponds to a pixel of distance value zero in the distance image. The actual matching is minimizing this edge distance. For the matching measure, one can choose median, arithmetic average, root mean square average (r.m.s.), or maximum.

An edge distance is computed for each position of the polygon, determined by the transformation equations. The position with the minimal edge distance is defined as the position with the best fit. The transformation equations that change the position of the polygon points should be parametric. Let $(\mathrm{x}, \mathrm{y})$ be the polygon coordinates and $(\mathrm{X}, \mathrm{Y})$ the position in the distance image. For example, for translation and rotation, the transformation equations become $\mathrm{X}=\mathrm{c}_{\mathrm{x}}+\mathrm{x} \cos \theta-\mathrm{y} \sin \theta$ and $\mathrm{Y}$ $=c_{y}+x \sin \theta+y \cos \theta$, where $\theta$ is the rotation angle, and $c_{x}$ and $c_{y}$ are the translation parameters in the $\mathrm{X}$ - and $\mathrm{Y}$-directions, respectively. Since the $(\mathrm{X}, \mathrm{Y})$ coordinates are not usually integers, they are frequently rounded to the nearest integer values.

Finding the optimal polygon position is equal to finding the global minimum of a multidimensional function. Thus, the minimization must be started very close to the optimal position to avoid falling in false local minima. For efficient search, a hierarchical matching algorithm is frequently used. If the minimum edge distance found is below a threshold (typically, $1-1.5$ ), it is considered that there is the model in the search image.

\subsection{Implementation}

Although there exist many methods for edge detection including the Canny edge detector [7], the Sobel edge operator [8] was used here for computational efficiency and good performance to extract the edge magnitude image.

Thresholding the edge gradient magnitude image obtained by applying the Sobel edge operator usually contains many thick edges. As the number of edges is increased, the matching speed gets slower. Thus, to reduce the number of edges, a nonmaxima suppression technique [9] was used for thinning edges, which suppresses all values not being the local maximum along the line of the gradient direction in the edge gradient magnitude image.

Fig. 1 shows an example of a model image and a search image, within which the model is searched. The search image was obtained by rotating by 12 degrees counterclockwise an image, from which Fig. 1(a) was extracted. Fig. 2 shows the edges extracted by applying the Sobel edge operator followed by the non-maxima suppression. Fig. 3 shows a distance image obtained by applying the 3-4 DT to Fig. 2(b). Edge pixels are completely black, and pixels are getting brighter as they get more distant from the edge pixels. 


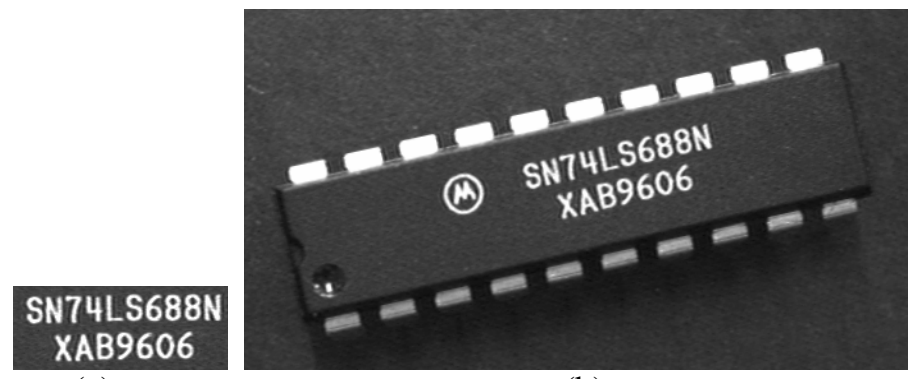

(a)

(b)

Fig. 1. (a) A model image. (b) A search image, within which the model is searched.

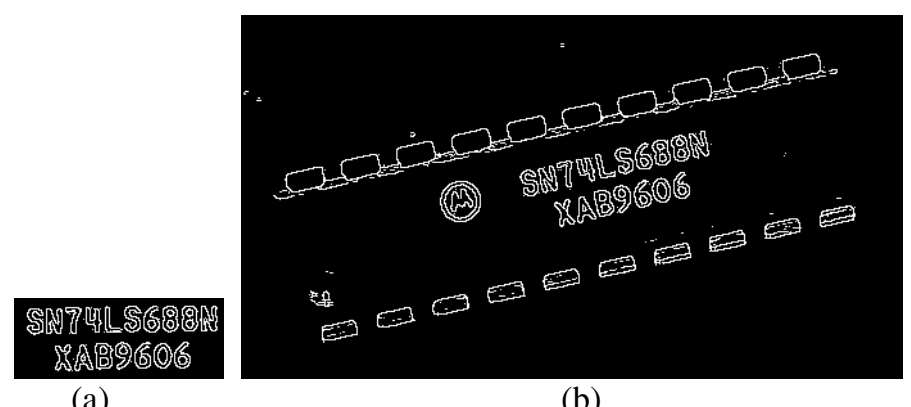

(a)

(b)

Fig. 2. (a) Edges of Fig. 1(a). (b) Edges of Fig. 1(b).

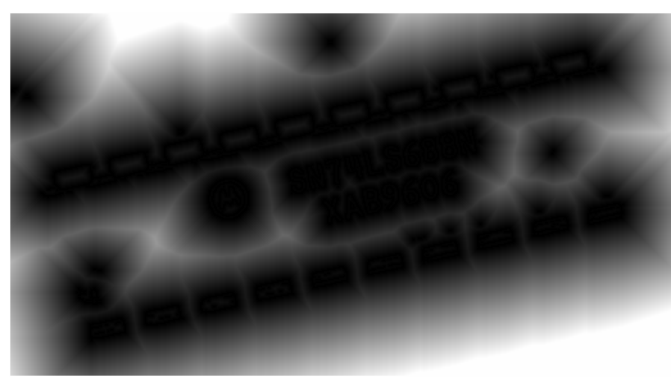

Fig. 3. A distance image of Fig. 2(b)

For the matching measure, among median, arithmetic average, root mean square average (r.m.s.), and maximum, r.m.s. was used here since it is known to give significantly fewer false minima than others [2]. The r.m.s. is given by

$$
d_{r m s}=\frac{1}{C} \sqrt{\frac{1}{n} \sum_{i=1}^{n} d_{i}^{2}},
$$

where $d_{i}$ is the distance value of the $i$-th point among $n$ points in the polygon, and $\mathrm{C}$ is a constant to compensate the unit distance in the DT. $(C=3$ for the $3-4$ DT, and $C=5$ for the 5-7-11 DT.) 
Start positions for chamfer matching are very important since they should be close to the optimal matching position to avoid falling in false local minima. Thus, the start positions were provided as outputs of a generalized Hough transform [10] for approximate matching positions. Here, three pose transformation parameters, translation and rotation, were assumed. Since in many industrial applications the appearance of the object to be found has limited degrees of freedom, rigid transformation (translation and rotation) is often sufficient.

Fig. 4 shows the results of matching the model of Fig. 2(a) with the edges of Fig 2(b); model edges are accurately overlaid on the test image, demonstrating that the matching position and angle were correctly found. (The positions of edges are denoted in black, and the white cross represents the reference point of the model, which is set to the center of the model image.)

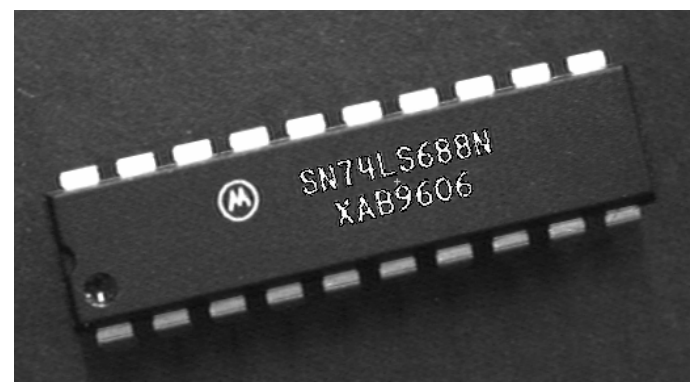

Fig. 4. The results of matching using the model of Fig. 2(a)

\subsection{An Improved Algorithm}

In computing the edge distance for each position of the polygon, determined by the transformation equation from the polygon coordinates $(\mathrm{x}, \mathrm{y})$ to the position $(\mathrm{X}, \mathrm{Y})$ in the distance image, the transformed coordinates $(\mathrm{X}, \mathrm{Y})$ are not usually integers, thus has been usually rounded to the nearest integer values.

However, for more accurate matching, we propose that the distance value at $(\mathrm{X}, \mathrm{Y})$ should be interpolated using adjacent pixels around $(\mathrm{X}, \mathrm{Y})$ of the distance image. Bilinear or bi-cubic interpolation can be used for this purpose. If bi-linear interpolation is used in the distance image $D$, the interpolated distance $D_{\text {xy }}$ at $(\mathrm{X}, \mathrm{Y})$ can be computed by

$$
\begin{array}{r}
D_{X Y}=(1-\alpha)(1-\beta) D(\lfloor X\rfloor,\lfloor Y\rfloor)+(1-\alpha) \beta D(\lfloor X\rfloor,\lfloor Y\rfloor+1) \\
+\alpha(1-\beta) D(\lfloor X\rfloor+1,\lfloor Y\rfloor)+\alpha \beta D(\lfloor X\rfloor+1,\lfloor Y\rfloor+1)
\end{array}
$$

where $\alpha=X-\lfloor X\rfloor, \beta=Y-\lfloor Y\rfloor$, and $\lfloor X\rfloor$ represents the truncated integer of $X$.

Table 1 shows the results of applying chamfer matching at the pixel level with/without bilinear interpolation in the distance image. We can notice that the distance value is considerably decreased by using the distance interpolation. 
Table 1. The results of applying chamfer matching

\begin{tabular}{|c|c|c|c|}
\hline & $\begin{array}{c}\text { Position [pi } \\
\text { xel] }\end{array}$ & $\begin{array}{c}\text { Angle } \\
\text { [degree] }\end{array}$ & Distance value \\
\hline Without distance interpolation & $(292,130)$ & 12 & 0.537 \\
\hline With distance interpolation & $(292,130)$ & 12 & 0.444 \\
\hline
\end{tabular}

To find more accurate matching parameters, a subpixel algorithm like a simple 1dimensional parabolic interpolation method can be used. Suppose some evaluation function $f(x)$ has the maximum value at discrete pixel value $x=H$, and let $f(H-1)=f_{1}$ and $\mathrm{f}(\mathrm{H}+1)=\mathrm{f}_{\mathrm{r}}$, then the locally maximum subpixel position $\mathrm{H}_{\mathrm{s}}$ can be estimated by the following parabolic interpolation:

$$
H_{s}=H+\frac{f_{r}-f_{i}}{2\left(2 f_{m}-f_{r}-f_{i}\right)}
$$

If the evaluation function has three parameters $\mathrm{x}, \mathrm{y}$, and angle, the 1-d interpolation method is applied independently for each parameter to estimate parameter values in subpixel. However, this method does estimate the matching position using interpolation without actual evaluation of the distance value in subpixel. Thus, a more accurate matching can be achieved by an optimization method with the interpolated distance evaluation for the minimum chamfer distance search. To this end, the Powell's method [6] may be used since it needs only the function evaluation without requiring function derivative computation.

Table 2 shows the results of applying the subpixel algorithms to Fig. 4 without the distance interpolation, and Table 3 shows the results of applying the subpixel

Table 2. The results of applying chamfer matching without the distance interpolation followed by the subpixel algorithm

\begin{tabular}{|c|c|c|c|}
\hline & Position[pixel] & Angle[degree] & Distance value \\
\hline Before subpixel algorithm & $(292,130)$ & 12 & 0.537 \\
\hline $\begin{array}{c}\text { After subpixel algorithm } \\
\text { (parabolic interpolation) }\end{array}$ & $(291.82,129.80)$ & 12.08 & 0.478 \\
\hline $\begin{array}{c}\text { After subpixel algorithm } \\
\text { (Powell) }\end{array}$ & $(291.76,129.73)$ & 12.00 & 0.463 \\
\hline
\end{tabular}

Table 3. The results of applying chamfer matching with the distance interpolation followed by the subpixel algorithm

\begin{tabular}{|c|c|c|c|}
\hline & Position[pixel] & Angle[degree] & Distance value \\
\hline Before subpixel algorithm & $(292,130)$ & 12 & 0.444 \\
\hline $\begin{array}{c}\text { After subpixel algorithm } \\
\text { (parabolic interpolation) }\end{array}$ & $(291.82,129.79)$ & 12.08 & 0.386 \\
\hline $\begin{array}{c}\text { After subpixel algorithm } \\
\text { (Powell) }\end{array}$ & $(291.72,129.70)$ & 12.00 & 0.378 \\
\hline
\end{tabular}


algorithms to Fig. 4 with the distance interpolation. We can notice that the distance value is further reduced by the subpixel algorithm. For the subpixel algorithm, the Powell's method seems to yield better results than the parabolic interpolation estimation.

\section{Accuracy Test of the Improved Algorithm}

The accuracy of the improved algorithm implemented was measured. First, to measure $\mathrm{x}, \mathrm{y}$ accuracy, an image containing Fig. 1(a) as a subimage, was translated in $\mathrm{x}$ and $\mathrm{y}$ axis direction in the range of $-0.8 \sim 0.8$ pixel by step size of 0.2 pixel, thus yielding 80 images in total. Using these images, the algorithm was run to obtain the translational accuracy (standard deviation and maximum error). Also, the test image was rotated within the range of $+/-30$ degrees by step size of 1 degree, and 60 images in total were obtained. Our algorithm was also run for these images to estimate the error of the rotation angle.

These results are shown in Table 4 and Table 5. (The model image used is Fig. 1(a), and bilinear interpolation was utilized in the translation and rotation of the image.) We can notice that the subpixel algorithm based on the parabolic interpolation yields $\mathrm{x}, \mathrm{y}$ accuracy of 0.14 pixel and angle accuracy of 0.15 degree in the worst case, while the Powell's method yields x accuracy of 0.06 pixel, y accuracy of 0.08 pixel, and angle accuracy of 0.04 degree in the worst case. Without the distance interpolation, the Powell's method for subpixel search gives poor results. The reason for this is that distance evaluation without interpolation should not be accurate due to insensitiveness to very small variations of $\mathrm{x}, \mathrm{y}$, and angle, thus being easily fallen into false local minima. Thus, the Powell's subpixel algorithm with the chamfer distance interpolation is found to be the most accurate.

The 3-4 DT was used throughout the above test, but we obtained nearly the same results with the 5-7-11 DT, known to be more accurate than the 3-4 DT. However, the 5-7-11 DT may be preferable to the 3-4 DT depending on the application.

Table 4. Accuracy of position and angle estimated without the chamfer distance interpolation

\begin{tabular}{|c|c|c|c|c|}
\hline $\begin{array}{c}\text { Subpixel } \\
\text { method }\end{array}$ & $\begin{array}{c}\mathrm{x} \\
\text { [pixel] }\end{array}$ & $\begin{array}{c}\mathrm{y} \\
\text { [pixel] }\end{array}$ & $\begin{array}{c}\text { angle } \\
\text { [degree] }\end{array}$ \\
\hline \multirow{2}{*}{$\begin{array}{c}\text { Parabolic } \\
\text { interpolation }\end{array}$} & Standard dev. & 0.09 & 0.09 & 0.07 \\
\cline { 2 - 5 } & Max. error & 0.14 & 0.12 & 0.19 \\
\hline $\begin{array}{c}\text { Powell's } \\
\text { optimization }\end{array}$ & Standard dev. & 0.30 & 0.32 & 0.06 \\
\cline { 2 - 5 } & Max. error & 0.42 & 0.71 & 0.31 \\
\hline
\end{tabular}

Table 5. Accuracy of position and angle estimated with the chamfer distance interpolation

\begin{tabular}{|c|c|c|c|c|}
\hline $\begin{array}{c}\text { Subpixel } \\
\text { method }\end{array}$ & $\begin{array}{c}\mathrm{x} \\
\text { [pixel] }\end{array}$ & $\begin{array}{c}\mathrm{y} \\
\text { [pixel] }\end{array}$ & $\begin{array}{c}\text { angle } \\
\text { [degree] }\end{array}$ \\
\hline \multirow{2}{*}{$\begin{array}{c}\text { Parabolic } \\
\text { interpolation }\end{array}$} & Standard dev. & 0.09 & 0.09 & 0.07 \\
\cline { 2 - 5 } & Max. error & 0.14 & 0.12 & 0.15 \\
\hline \multirow{2}{*}{$\begin{array}{c}\text { Powell's } \\
\text { optimization }\end{array}$} & Standard dev. & 0.03 & 0.03 & 0.02 \\
\cline { 2 - 5 } & Max. error & 0.06 & 0.08 & 0.04 \\
\hline
\end{tabular}




\section{Conclusion}

In this paper, an improved approach for accurate chamfer matching was presented that uses interpolation in the distance calculation for subpixel distance evaluation. For more accurate matching, instead of estimating the optimal position in subpixel using neighborhood of the pixel position with the minimum distance, we used the Powell's optimization to find the distance minimum through actual distance evaluations in subpixel. Experimental results demonstrated the effectiveness of our approach.

\section{References}

1. Barrow, H.G., Tenenbaum, J.M., Bolles, R.C., Wolf, H.C.: Parametric correspondence and chamfer matching: Two new techniques for image matching. In: Proc. 5th Int. Joint Conf. Artificial Intelligence, Cambridge, MA, pp. 659-663 (1977)

2. Borgefors, G.: Hierarchical chamfer matching: a parametric edge matching algorithm. IEEE Trans. Pattern Analysis and Machine Intelligence 10(6), 849-865 (1988)

3. Chetverikov, D., Khenokh, Y.: Matching for Shape Defect Detection. In: Solina, F., Leonardis, A. (eds.) CAIP 1999. LNCS, vol. 1689, pp. 367-374. Springer, Heidelberg (1999)

4. Gavila, D.: Pedestrian detection from a moving vehicle. In: Vernon, D. (ed.) ECCV 2000. LNCS, vol. 1842, pp. 37-49. Springer, Heidelberg (2000)

5. Thayananthan, A., Stenger, B., Torr, P.H.S., Cipolla, R.: Shape context and chamfer matching in cluttered scenes. In: Proc. CVPR 2003, Madison, Wisconsin, pp. 127-135 (2003)

6. Press, W.H., Flannery, B.P., Teukolsky, S.A., Vetterling, W.T.: Numerical Recipes in C, 2nd edn. Cambridge University Press, Cambridge (1992)

7. Canny, J.: A computational approach to edge detection. IEEE Trans. Pattern Analy. and Mach. Intelli. 8(6), 679-698 (1986)

8. Davies, E.R.: Machine Vision, 3rd edn. Morgan Kaufmann, San Francisco (2005)

9. Jain, R., Kasturi, R., Schunck, B.G.: Machine Vision. McGraw-Hill, New York (1995)

10. Ballard, D.H.: Generalizing Hough transform to detect arbitrary shapes. Pattern Recognition 13(2), 111-122 (1981)

11. Borgefos, G.: Distance transformations in digital images. Computer Vision, Graphics, and Image Processing 34(3), 344-371 (1986) 